\title{
Syphilis: presentations in general medicine
}

\author{
Authors: Farai Nyatsanza ${ }^{A}$ and Craig Tipple ${ }^{B}$
}

Syphilis is caused by the spirochete bacterium Treponema pallidum and can be transmitted both sexually and from mother to child. $T$ pallidum can infect any organ and produces a clinical disease with a relapsing and remitting course. It is not hard to see, therefore, why it is often described as the great mimic. In this review, we provide an update of modern syphilis epidemiology, clinical presentations, and testing and treatment strategies.

\section{Introduction}

Syphilis is caused by the bacterium Treponema pallidum subspecies pallidum . ${ }^{1}$ This motile, gram-negative spirochete can be transmitted both sexually and from mother to child, and can invade virtually any organ or structure in the human body. The disease, which should be considered systemic throughout, is characterised by overlapping clinical stages and a relapsing and remitting course. This potentially diverse presentation led Sir William Osler to call syphilis 'the great imitator' and, together with a greatly increased incidence over the last 15 years, explains its importance to the general physician.

\section{Epidemiology}

Syphilis emerged in Europe after 1492 following the return of Columbus from the New World, although its presence in Europe prior to his return is still debated. Paleontological findings have been joined by data from gene sequence analysis of T pallidum subspecies and strains to both support and refute the 'Columbian' theory. ${ }^{1,2}$ No matter the origin, syphilis spread rapidly through Europe in the 15th century. By the end of Queen Victoria's reign, around 1:10 sexually active adults in London were thought to be infected, although the true prevalence wasn't known until the description of the first serologic test by Auguste Wasserman in $1906 .^{3}$

Incidence in the UK declined greatly following the widespread use of penicillin in the early 1950s. Unfortunately, the last 15 years have seen a 10 -fold increase in cases, with 4,317 reported

Authors: ${ }^{A}$ specialist trainee in genitourinary medicine, Jefferiss Wing for Sexual Health, Imperial College NHS Healthcare Trust, London, UK; ${ }^{B}$ consultant physician in genitourinary medicine, Jefferiss Wing for Sexual Health, Imperial College NHS Healthcare Trust, London, UK new infections in 2014 - the highest for over 40 years. This rise is predominantly among men who have sex with men (MSM), who account for $80.6 \%$ of cases. ${ }^{4}$ Enhanced surveillance data show that the general profile is one of white MSM aged 25-34, reporting high numbers of sexual partners, condomless sex, recreational drug use and the use of social networking apps to find sexual partners. Approximately $40 \%$ are co-infected with HIV-1. ${ }^{5}$ Over a similar period, there has been a decline of $16 \%$ in female cases from 317 in 2003 to 265 in 2012. In 2011, the overall incidence of congenital syphilis in England was $0.0025 / 1,000$ births. Cases predominated among women not accessing healthcare due to cultural barriers and social deprivation. ${ }^{6}$

\section{Clinical presentation}

The clinical course of syphilis is one of overlapping clinical stages which begin 9-90 (median 21) days following direct contact with an infectious lesion (Fig 1).

\section{Primary syphilis}

Primary syphilis is characterised by a papule at the point of entry of the bacterium that breaks down into an ulcer (chancre). Although classically anogenital, it can be in the mouth $(30 \%$ of cases are transmitted through orogenital contact), rectum, cervix or other such clinically 'quieter' locations. A chancre is typically painless, $0.5-2 \mathrm{~cm}$ in diameter, firm or rubbery, and associated with regional lymphadenopathy (Fig 2). It usually heals over a 4-6 week period. Atypical presentations with multiple or painful ulcers can occur, especially in the context of HIV-1 co-infection. ${ }^{7}$ Important differential diagnoses are herpes simplex virus and lymphogranuloma venerum.

\section{Secondary syphilis}

The secondary stage of infection begins 4-10 weeks after the ulcer has healed, although this is highly variable and the primary and secondary stages can be concurrent. The hallmark sign is a maculopapular rash (seen in $50-70 \%$ of patients) that may affect the palms and soles (Fig 2). ${ }^{8}$ Other signs and symptoms are described in Table 1 . The rash and especially the moist lesions of secondary syphilis are infectious. Given the shared epidemiology of HIV and syphilis, primary HIV infection is an important differential diagnosis in MSM presenting with a rash. 


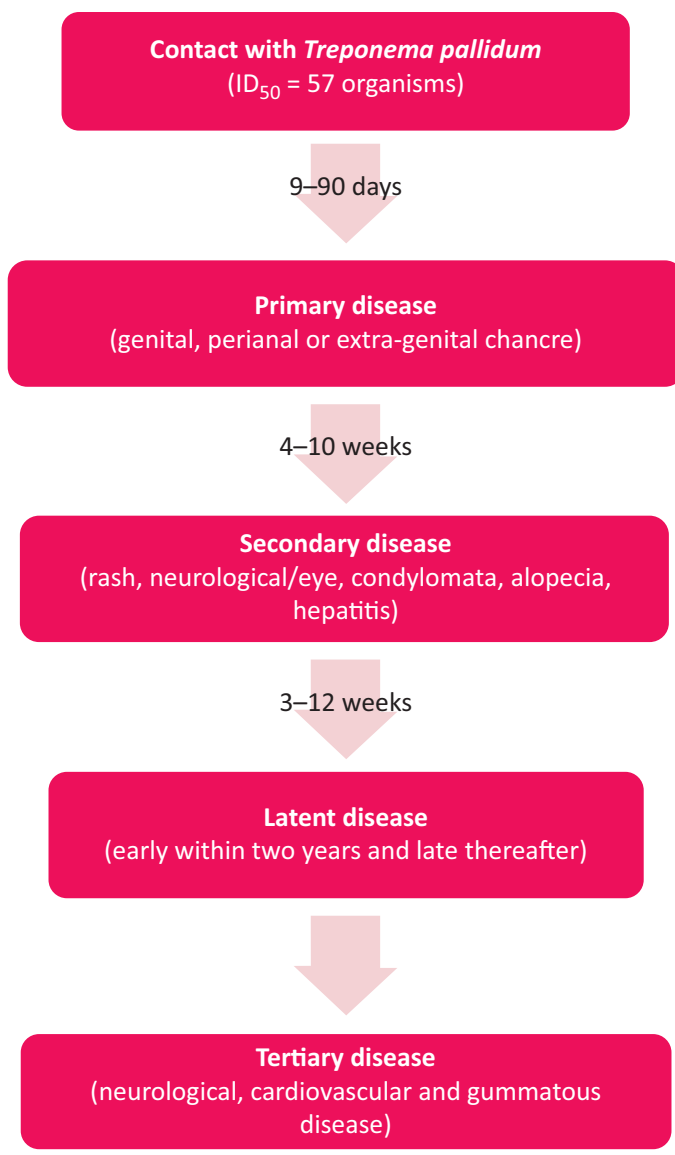

Fig 1. The clinical stages of syphilis. The clinical stages of syphilis from contact with $T$ pallidum through to development of tertiary disease. During the first year of latent infection, $25 \%$ of patients will relapse to the secondary stage.

\section{Latent syphilis}

In the absence of treatment, features of secondary syphilis typically regress within three months and the disease becomes latent and non-infectious. Clinical relapses may occur during the first two years of latency (early latent disease), but are rare thereafter (late latent disease). ${ }^{9}$

\section{Tertiary disease}

Following the latent stage (typically 15-30 years), signs and symptoms of tertiary infection may occur. These are now rare due to the lower prevalence of the disease 15-30 years ago and the widespread use of treponemocidal and treponemostatic antibiotics (penicillins, tetracyclines, macrolides, cephalosporins) for other infections. Tertiary disease is typically divided into gummatous (the most common), cardiovascular and neurological disease. Deep-seated and destructive gumma can occur in any organ but mainly affect the skin and bones. Cardiovascular syphilis mainly affects the aortic valve and ascending aorta most commonly causing aortic dilatation and regurgitation. Neurological features are discussed below.

\section{Neurological involvement}

Neurological involvement can occur at any stage. During the early stages of syphilis, the meninges, vasculature, cranial nerves (particularly II and VIII) and the eyes are most commonly affected. In the tertiary stage, the most common forms involve the brain and spinal cord parenchyma. Each form has characteristic clinical findings, although some overlap may occur (Table 1). All patients with suspected or confirmed syphilis and neurological symptoms should undergo a full neurological examination.?

\section{Testing}

Serologic tests can be used to diagnose all stages of syphilis. They consist of treponemal tests (TT) such as the Treponema pallidum particle agglutination (TPPA) assay or the IgM/IgG enzyme immunoassay (EIA) and non-treponemal (anticardiolipin) tests (NTT) such as rapid plasma reagin (RPR) or venereal disease research laboratory (VDRL) tests (NB VDRL testing is no longer widely available in the UK). ${ }^{10}$ TT are often the first to become positive (from two weeks after infection) and usually remain positive for life. NTT are performed
Fig 2. Clinical pictures of patients with early syphilis. Clinical photographs of patients with early syphilis: a) typical maculopapular rash on the chest; b) skin with secondary syphilis rash at high magnification; c) penile chancre. Images obtained from the Wellcome Photo Library (N0000562, N0000822 and N0000823) and reproduced with permission.
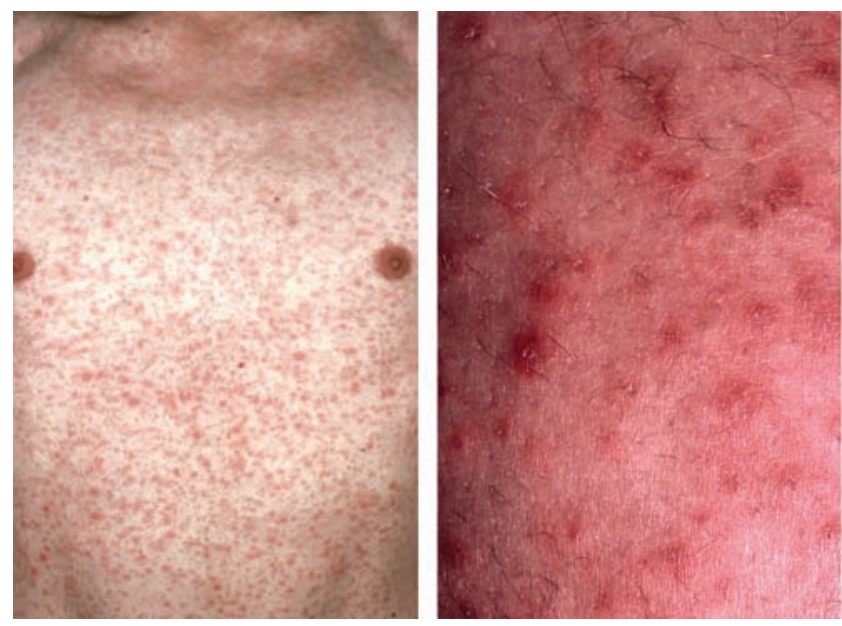
Table 1. The clinical manifestations of syphilis.

\author{
Stage of disease Signs and symptoms \\ Secondary Systemic: \\ syphilis > myalgia \\ $>$ fever \\ weight loss \\ rash: maculo-papular (50-70\%), papular (12\%), macular (10\%), annular (6-14\%); rarely: framboiseform, lues \\ maligna \\ alopecia $(4-11 \%)$ \\ generalised lymphadenopathy ( $85 \%)$. \\ Local: \\ > condylomata lata (10\%): wart-like lesions which affect moist intertriginous areas (peri-anal, vulval, groin, scrotum) \\ subclinical hepatitis (10\%) (a mild transaminitis is common) \\ renal (immune complex-mediated glomerulonephritis) (rare) \\ neurological involvement: symptomatic meningitis, infectious arteritis (ischaemia, thrombosis, infarction), \\ otosyphilis (hearing loss with or without tinnitus), ocular disease (28-51\% of neurosyphilis) (anterior and \\ posterior uveitis, iritis, chorioretinitis, necrotizing retinitis and optic neuritis).
}

Neurosyphilis Asymptomatic - early/late: Abnormal CSF with no signs/symptoms. This is of uncertain significance as CSF abnormalities have been found in up to $70 \%$ of primary and secondary syphilis and less than $10 \%$ will develop disease.

Meningovascular (2-7 years): Focal arteritis inducing infarction/meningeal inflammation; signs dependent on site of vascular insult. Occasional prodrome; headache, emotional lability, insomnia.

General paresis (10-20 years): Cortical neuronal loss causing gradual decline in memory and cognitive functions. Emotional lability, personality change, psychosis and dementia. Seizures and hemiparesis are late complications.

Tabes dorsalis: Inflammation of spinal dorsal column/nerve roots; lightening pains, areflexia, paraesthesia, sensory ataxia, Charcot's joints, optic atrophy, pupillary changes (eg Argyll Robertson pupil).

$\mathrm{CSF}=$ cerebrospinal fluid

\section{Key points}

The incidence of syphilis has increased significantly over the last 15 years. The majority of cases are among men who have sex with men (MSM) aged $25-35$ of whom $40 \%$ are HIV positive.

Syphilis is a systemic disease from the outset and can involve any organ. It typically presents with a genital ulcer or a rash but its manifestations are protean.

For MSM presenting unwell with a rash, primary HIV infection is a key differential diagnosis and all patients with suspected syphilis should also be tested for HIV.

Diagnosis is confirmed serologically using two specific treponemal tests followed by a non-treponemal test to assess disease stage and to allow treatment monitoring.

Penicillin G treatment remains effective as does oral doxycycline, but macrolide antibiotics should be avoided due to widespread resistance.

KEYWORDS: Syphilis, Treponema pallidum, epidemiology, treatment, neurosyphilis quantitatively and are used to monitor treatment response. They give an indication of disease stage as higher titres are associated with more active (early) infection and lower titres indicate quieter (latent) or previously treated infection. NTT are subject to both biologic false positive (pregnancy, recent vaccination, autoimmune disease) and false negative (at very high titres due to a prozone phenomenon) results. It is also important to note that NTT may be negative in early primary infection.

There are two testing algorithms in use termed 'conventional' and 'reverse'. The reverse algorithm is used in the UK and begins with an EIA followed by confirmation with a second TT (TPPA). Stage of disease is then assessed with the RPR titre. ${ }^{11}$ This method has the advantage of an automatable screening test (EIA) but can have a lower positive predictive value in low prevalence populations. It is noteworthy that other treponemal infections (yaws, pinta, bejel) are serologically indistinct from venereal syphilis.

Serologic tests can be performed on cerebrospinal fluid (CSF). A positive NTT is highly specific for neurosyphilis but lacks sensitivity. A negative TT on a CSF sample with a negligible red blood cell count (visible blood contamination can lead to false positive results) effectively excludes neurosyphilis.

In the genitourinary medicine (GUM) clinic, primary and secondary syphilis can also be diagnosed by the direct identification of T pallidum using darkground microscopy 
(DGM) or polymerase chain reaction (PCR). ${ }^{10}$ With optimal conditions, DGM can have a sensitivity of $80 \% .^{12}$ Due to contamination by commensal treponemes, such as Treponema denticola, the specificity of DGM for oral and rectal lesions is low. T pallidum PCR has a characteristically high sensitivity $(80-100 \%)$ and specificity $(92.1-99.8 \%) .^{13}$

\section{Management}

Patients with signs or symptoms of syphilis should be referred to a GUM physician. The current recommended antimicrobial treatment of syphilis is well explained in the national treatment guideline. ${ }^{9}$ Basic management considerations are as follows.

Benzylpenicillin (penicillin G) was first used to treat syphilis in 1943 and penicillin is still the first-line treatment for all stages of syphilis. ${ }^{9}$ Duration and route vary according to stage but in the absence of neurological involvement depot parental preparations of penicillin are first line. In patients with allergy, doxycycline and ceftriaxone are alternative treatments.

$>$ Patients with syphilis are at risk of other sexually transmitted infections (STIs). A full sexual history should be taken and STI screening, including an HIV test, should be offered.

> An RPR titre should be sent on the first day of treatment as a four-fold (two dilution) reduction in titre is the established serologic measure of treatment success.

> Patients should abstain from sexual contact for two weeks following treatment for early syphilis. ${ }^{15}$ All sexual contacts within the last three months should be contacted and tested. HIV-1 infected patients with CD 4 counts $<350$ cells/ml and/ or RPR titres $\geq 1: 32$ may be at increased risk of neurological involvement.

> Patients with symptomatic disease should be warned about Jarisch-Herxheimer reaction (JHR) prior to treatment. The JHR is a self-limiting acute febrile illness which typically begins 12 hours after treatment and is complete 12 hours later. ${ }^{9}$ It is a particular concern in pregnancy and in patients with neurological or cardiovascular involvement. ${ }^{16}$ Steroids can be given before and during the first few days of treatment for neurological and cardiovascular syphilis. In most cases, rest and paracetamol suffice.

\section{Antimicrobial resistance}

Despite over 70 years of use, $T$ pallidum remains sensitive to penicillin. ${ }^{17}$ Azithromycin was proven an effective treatment for early disease in two randomised controlled trials. ${ }^{18,19}$ Unfortunately, $60-80 \%$ of T pallidum strains in the UK are macrolide resistant thus it cannot be used reliably. ${ }^{20}$ As yet, there has been no description of tetracycline resistance in Tpallidum.

\section{Managing syphilis: a worked example}

A 78 year-old Jamaican gentleman with memory impairment had syphilis serology sent as part of a dementia screen. The result returned as EIA positive, TPPA positive and RPR negative.

\section{Key history}

1. Where possible, take a sexual history in a confidential environment. ${ }^{14}$

2. Previous syphilis or syphilis treatment?

o If yes, ascertain if this treatment was adequate (compliance, treatment given, partners treated?).

3. Previous syphilis testing? (NB For a female patient, syphilis testing may have been performed during antenatal screening).

o If previously negative, then this could be new infection.

4. History of yaws (T pallidum subspecies pertenue) infection? o If yes, then serologic results may be secondary to yaws. If there is a clear history of treatment and the RPR is negative then patient may not require further treatment.

5. Previous symptoms consistent with primary or secondary syphilis? Current symptoms consistent with tertiary disease (other than memory impairment)?

\section{Examination}

> Seek other signs of late neurosyphilis in addition to gummatous and cardiovascular disease. Be mindful of the possibility of late congenital syphilis, signs of which are detailed elsewhere. ${ }^{9}$

\section{Management}

$>$ Liaise with GUM team.

Relevant neurological signs should prompt CSF examination (with prior brain imaging such as CT or $\mathrm{MRI}$ ). If the patient cannot tolerate lumbar puncture, then consider presumptive neurosyphilis treatment. This patient had a negative serum RPR, thus late neurosyphilis is highly unlikely.

$>$ Offer screening to past and present sexual partners (if contactable) and children if there is no evidence of the mother of the children being tested.

\section{Conclusion}

Syphilis has re-emerged as an important sexually transmitted infection. Timely diagnosis and treatment is necessary to prevent onward transmission and the development of irreversible tissue damage. Suspicion of the infection should prompt serologic testing and referral to a GUM physician.

\section{Acknowledgements}

The authors thank Dr David Goldmeier for his helpful input during the preparation of this manuscript.

\section{References}

1 Radolf J, Lukehart S. Pathogenic Treponema: molecular and cellular biology. Haverhill: Caister Academic Press, 2006.

2 Smajs D, Norris SJ, Weinstock GM. Genetic diversity in Treponema pallidum: implications for pathogenesis, evolution and molecular diagnostics of syphilis and yaws. Infect Genet Evol 2012;12:191-202.

3 Wasserman A, Neisser F, Bruck C. Eine serodiagnostische Reaktion bei Syphilis. Deutsche medicinische Wochenschrift 1906;32:745-6.

4 Public Health England. Sexually transmitted infections and chlamydia screening in England 2014. Health Protection Report 
2014;9:22-9. Available online at www.gov.uk/government/uploads/ system/uploads/attachment_data/file/437433/hpr2215_STI_NCSP_ v6.pdf [Accessed 20 January 2016].

5 Health Protection Agency. Syphilis and lymphogranuloma venereum: resurgent sexually transmitted infections in the UK: 2009 report. London: Health Protection Agency, 2009.

6 Public Health England. Recent epidemiology of infectious syphilis and congenital syphilis. Infection Reports 2013;7(44). Available online at www.gov.uk/government/uploads/system/uploads/attachment_data/file/336760/hpr4413_sphls.pdf [Accessed 20 January 2016].

7 Tipple C. Impact of HIV-1 infection on the clinical presentation of syphilis in men who have sex with men. Sex Health 2014;12:110-8.

8 Baughn RE, Musher DM. Secondary syphilitic lesions. Clin Microbiol Rev 2005;18:205-16.

9 Kingston M, French P, Goh B et al. UK National Guidelines on the Management of Syphilis 2008. Int J STD AIDS 2008;19:729-40.

10 Larsen S, Steiner B, Rudolph A. Laboratory diagnosis and interpretation of tests for syphilis. Clin Microbiol Rev 1995;8:1-21.

11 Public Health England. UK Standards for Microbiology Investigations (SMIs) V 44: Syphilis serology. London: PHE, 2014. Available online at www.gov.uk/government/publications/smi-v-44-serologicaldiagnosis-of-syphilis [Accessed 20 January 2016].

12 Wheeler HL, Agarwal S, Goh BT. Dark ground microscopy and treponemal serological tests in the diagnosis of early syphilis. Sex Transm Infect 2004;80:411-4.

13 Palmer H, Higgins S, Herring A, Kingston M. Use of PCR in the diagnosis of early syphilis in the United Kingdom. Sex Transm Infect 2003;79:479-83.
14 Brook G, Bacon L, Evans C et al. 2013 UK national guideline for consultations requiring sexual history taking. Clinical Effectiveness Group British Association for Sexual Health and HIV. Int J STD AIDS 2014;25:391-404.

15 Workowski KA, Bolan GA, CfDCa Prevention. Sexually transmitted diseases treatment guidelines, 2015. MMWR Recomm Rep 2015;64:1-137.

16 Klein VR, Cox SM, Mitchell MD, Wendel GD. The JarischHerxheimer reaction complicating syphilotherapy in pregnancy. Obstet Gynecol 1990;75:375-80.

17 Stamm LV. Global challenge of antibiotic-resistant Treponema pallidum. Antimicrob Agents Chemother 2010;54:583-9.

18 Riedner G, Rusizoka M, Todd J et al. Single-dose azithromycin versus penicillin G benzathine for the treatment of early syphilis. $N$ Engl J Med 2005;353:1236-44.

19 Hook EW, Behets F, Van Damme K et al. A phase III equivalence trial of azithromycin versus benzathine penicillin for treatment of early syphilis. J Infect Dis 2010;201:1729-35.

20 Tipple C, McClure MO, Taylor GP. High prevalence of macrolide resistant Treponema pallidum strains in a London centre. Sex Transm Infect 2011;87:486-8.

Address for correspondence: Dr C Tipple, Jefferiss Wing for Sexual Health, Imperial College Healthcare NHS Trust, St Marys Hospital, Praed Street, London W2 1NY, UK. Email: craig.tipple@imperial.nhs.uk

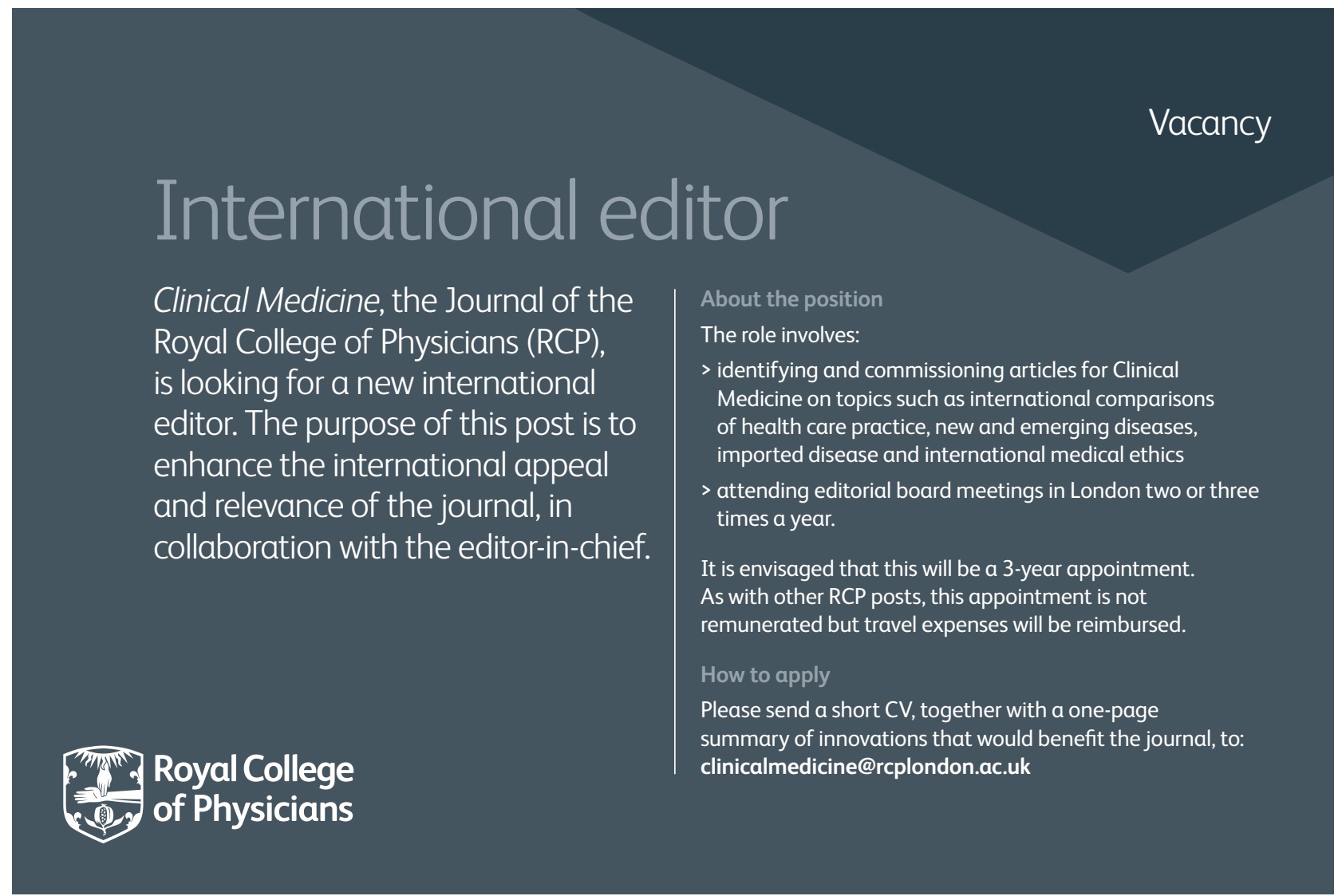

